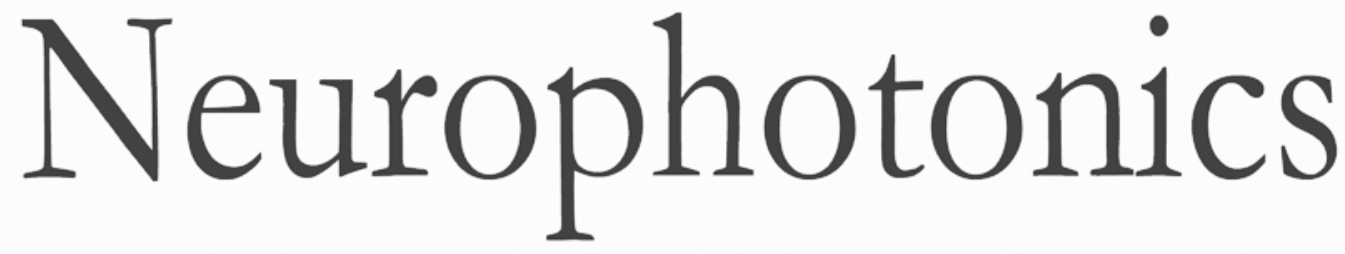

\title{
Single nanoparticle tracking of $N$-methyl-D-aspartate receptors in cultured and intact brain tissue
}

\author{
Juan A. Varela \\ Joana S. Ferreira \\ Julien P. Dupuis \\ Pauline Durand \\ Delphine Bouchet \\ Laurent Groc
}




\title{
Single nanoparticle tracking of $\mathbf{N}$-methyl-D-aspartate receptors in cultured and intact brain tissue
}

\author{
Juan A. Varela, ${ }^{a, b, \dagger}$ Joana S. Ferreira, ${ }^{a, b, \dagger}$ Julien P. Dupuis, ${ }^{a, b, \dagger}$ Pauline Durand, ${ }^{a, b}$ Delphine Bouchet, ${ }^{a, b}$ and \\ Laurent Groc ${ }^{\mathrm{a}, \mathrm{b}, *}$ \\ aUniversité de Bordeaux, Interdisciplinary Institute for Neuroscience, UMR 5297, 146 rue Leo Saignat, 33077 Bordeaux, France \\ ${ }^{\mathrm{b}}$ Centre National de la Recherche Scientifique, Interdisciplinary Institute for Neuroscience, UMR 5297, 33000 Bordeaux, France
}

\begin{abstract}
Recent developments in single-molecule imaging have revealed many biological mechanisms, providing high spatial and temporal resolution maps of molecular events. In neurobiology, these techniques unveiled that plasma membrane neurotransmitter receptors and transporters laterally diffuse at the surface of cultured brain cells. The photostability of bright nanoprobes, such as quantum dots (QDs), has given access to neurotransmitter receptor tracking over long periods of time with a high spatial resolution. However, our knowledge has been restricted to cultured systems, i.e., neurons and organotypic slices, therefore lacking several aspects of the intact brain rheology and connectivity. Here, we used QDs to track single glutamatergic $\mathrm{N}$-methyl-D-aspartate receptors (NMDAR) in acute brain slices. By delivering functionalized nanoparticles in vivo through intraventricular injections to rats expressing genetically engineered-tagged NMDAR, we successfully tracked the receptors in native brain tissue. Comparing NMDAR tracking to different classical brain preparations (acute brain slices, cultured organotypic brain slices, and cultured neurons) revealed that the surface diffusion properties shared several features and are also influenced by the nature of the extracellular environment. Together, we describe the experimental procedures to track plasma membrane NMDAR in dissociated and native brain tissue, paving the way for investigations aiming at characterizing receptor diffusion biophysics in intact tissue and exploring the physiopathological roles of receptor surface dynamics. $\odot$ The Authors. Published by SPIE under a Creative Commons Attribution 3.0 Unported License. Distribution or reproduction of this work in whole or in part requires full attribution of the original publication, including its DOI. [DOI: 10.1117/1.NPh.3.4.041808]
\end{abstract}

Keywords: quantum dot; single-molecule tracking; lateral diffusion; glutamate.

Paper 16019SSR received Mar. 24, 2016; accepted for publication Jun. 22, 2016; published online Jul. 12, 2016.

\section{Introduction}

Over the past years, neurobiology has experienced a genuine revolution as microscopy techniques made it possible to image single molecules in live biological environments. ${ }^{1-4}$ This was particularly relevant to understand the complex organization of synapses ( 200 to $400 \mathrm{~nm}$ in diameter) for which the use of light microscopy imaging was strongly limited by diffraction. Singlemolecule imaging approaches have provided the first set of tools to study in live neurons the molecular organization and dynamics of synaptic components such as neurotransmitter receptors. ${ }^{5,6}$ Compared to classical bulk fluorescence approaches that allow ensemble measurements, single-molecule tracking techniques also have the advantage of extracting the characteristics of different subpopulations that may have specific biological functions. The different techniques that have been used to achieve single-molecule tracking in cells rely on the lighting up of very few bright molecules at a given time to resolve single emitting objects in a noisy biological background. Some of these techniques are based on the "photoswitching" (e.g., photoactivated localization microscopy) or bleaching (e.g., universal point accumulation for imaging in nanoscale topography) of individual fluorophores, or on the imaging of photostable nanoparticles. ${ }^{7-9}$ Each technique has advantages and drawbacks, and the choice generally depends on the biological question and

*Address all correspondence to: Laurent Groc, E-mail: laurent.groc@ u-bordeaux.fr

†Theses authors contributed equally to this work. accessibility of the molecule of interest. For instance, long trajectories of a single molecule may be required to tackle a biological question, orienting the choice toward photostable nanoparticles. The most commonly used nanoparticles to perform single molecule are semiconductor nanocrystals called quantum dots (QDs). The nanocrystals can be composed of various elements, such as a core of CdSe beneath a shell of $\mathrm{ZnS} .^{10}$ Due to their relatively small size (5 to $10 \mathrm{~nm}$ ), large Stokes shift, narrow emission spectrum, brightness (an order of magnitude more than the brightness of an organic dye), and photostability, ${ }^{10}$ they have been extensively used over the past few years to label a large variety of biomolecules; in particular in the field of neuroscience QDs helped disclose unexpected mechanisms regulating synaptic transmission.

Indeed, single-molecule imaging has shed new light on neurotransmitter receptor organization and trafficking at the plasma membrane of cultured brain cells. All neurotransmitter receptors examined so far have been found to be highly dynamic at the surface of astrocytes and neurons, where they exchange between synaptic and extrasynaptic compartments. ${ }^{1-13}$ Using single-molecule approaches, differences in molecular behaviors have been uncovered among and within families of receptors including the excitatory glutamate alpha-amino-3-hydroxy-5methylisoazol-4-propionate and $N$-methyl-D-aspartate (NMDA) receptors, inhibitory glycinergic and $\mathrm{GABA}_{\mathrm{A}}$ receptors, and modulatory dopamine receptors. ${ }^{7,14-17}$ Noteworthy, similar outcomes have been obtained when exploring the surface dynamics of neurotransmitter transporters in neurons and astrocytes (glutamate transporter GLT1 and dopamine transporter). ${ }^{18,19}$ Among 
the glutamatergic receptors, NMDA receptors (NMDAR) have attracted much attention since they play a key role in synaptic plasticity processes, network maturation, cognitive functions, and because impairments in NMDAR signaling have been associated with severe neuropsychiatric conditions. ${ }^{20}$ Revealing the rules that govern the surface and intracellular trafficking of NMDAR has thus emerged as a central aim to understand the physiology of neuronal networks and the mechanisms leading to brain disorders. ${ }^{21}$

Single-molecule tracking studies have been largely restricted to cultured brain cells (neurons and astrocytes) in which an optimal spatial and temporal control of the probes can be achieved. However, the biological architecture of the native environment in which neural processes take place is much more complex than what can be accessed through dissociated cultures. A step toward integrated systems has been recently taken by tracking single molecules in live cultured organotypic brain slices. ${ }^{22}$ Both cultured neurons and organotypic slices, however, lack key characteristics of the intact brain such as dendritic morphology, topography of axonal inputs, and complexity of the extracellular environment, which are likely to influence the distribution and dynamics of surface receptors but are accessible only in or ex vivo. Imaging neurotransmitter receptors at the single-molecule level in native brain preparations are thus fundamental. Here, we describe how to perform single QDs tracking in various brain network preparations, including freshly dissected acute brain slices, and draw comparisons among NMDAR diffusion properties in these experimental configurations.

\section{Methods}

We used three different neurobiological preparations to perform single QDs tracking in neurons, as described below.

\subsection{Single Quantum Dots Tracking in Acute Brain Slices}

\subsubsection{Postnatal electroporation}

Sprague-Dawley rats (Janvier, France) were used for this work, both male and female, treated according to the guidelines of the University of Bordeaux/Centre National de la Recherche Scientifique Animal Care and Use Committee. Electroporation was done in newborn pups, between postnatal day $(\mathrm{P})$ zero (P0) and P1, as previously described. ${ }^{23}$ Pups were anesthetized by hypothermia and injected with a mixture of two deoxyribose nucleic acid (DNA) constructs, coding for GluN1super-ecliptic pHluorin (SEP) and cytosolic enhanced green fluorescent protein (EGFP) to identify transfected cells. Approximately $2 \mu \mathrm{g}$ of DNA in $8 \mu \mathrm{l}$ of phosphate-buffered saline (PBS) and $1 \mu \mathrm{l}$ of Fast Green were injected in both lateral ventricles. The injection point was obtained by drawing an imaginary line between the eye and lambda, identifying the midpoint, and retroceding $2 \mathrm{~mm}$ from that midpoint. The injection was performed at a depth of $2.6 \mathrm{~mm}$ under cold illumination. The success of the injection was evaluated by the ventricular dispersion of the Fast Green dye. Successfully injected pups were electroporated using forceps-type circular electrodes (7mm platinum Tweezertrode BTX, Harvard apparatus) spread with conductive gel. Electrodes were positioned either with the positive pole under the throat and the negative on top of the head to electroporate hippocampal neurons, or the opposite to electroporate cortical neurons. Five electrical pulses (150 V, 50-ms duration, 1-s interval between pulses) were delivered with a pulse generator (BTX Harvard apparatus ECM830), and pups were immediately reanimated on a thermal blanket at $37^{\circ} \mathrm{C}$ before being placed back with the mother.

\subsubsection{Quantum dot injection}

To prepare QDs dispersions, $2 \mu \mathrm{l}$ of QD655 goat F(ab')2 antirabbit $\mathrm{IgG}$ (Invitrogen, \#Q-11421MP) or antimouse IgG (Invitrogen, \#Q11022MP) were mixed with $2 \mu$ of GFP rabbit serum polyclonal antibody (Molecular Probes, \#A6455) or anti-GFP mouse $\operatorname{IgG} 1 \kappa$ (clones 7.1 and 13.1, ROCHE, \#11814460001) and $26 \mu \mathrm{l}$ of PBS. After $30 \mathrm{~min}$, two previously electroporated P4 pups were anesthetized by hypothermia and injected with QDs solutions in the lateral ventricles $(\sim 15 \mu \mathrm{l}$ per pup). The coordinates were obtained as described above, except that the depth was $3.5 \mathrm{~mm}$ for $\mathrm{P} 4$ pups. After reanimation on a heating blanket, the pups were placed back with their mother.

\subsubsection{Acute brain slices}

Postnatal day $\mathrm{P} 4$ rats that were injected with QDs were sacrificed for 1.5 to $2 \mathrm{~h}$ after QDs injection, and parasagittal brain slices (350- $\mu \mathrm{m}$ thick) were prepared in an ice-cold sucrose buffer solution containing (in $\mathrm{mM}$ ): 250 sucrose, $2 \mathrm{KCl}, 7 \mathrm{MgCl}_{2}, 0.5 \mathrm{CaCl}_{2}$, $1.15 \mathrm{NaH}_{2} \mathrm{PO}_{4}, 11$ glucose, and $26 \mathrm{NaHCO}_{3}$ (gassed with $95 \% \mathrm{O}_{2} / 5 \% \mathrm{CO}_{2}$ ). Slices were then incubated for $30 \mathrm{~min}$ at $33^{\circ}$ $\mathrm{C}$ and subsequently stored at room temperature in an artificial cerebrospinal fluid (ACSF) solution containing (in $\mathrm{mM}$ ): $126 \mathrm{NaCl}$, $3.5 \mathrm{KCl}, 2 \mathrm{CaCl}_{2}, 1.3 \mathrm{MgCl}_{2}, 1.2 \mathrm{NaH}_{2} \mathrm{PO}_{4}, 25 \mathrm{NaHCO}_{3}$, and 12.1 glucose (gassed with $95 \% \mathrm{O}_{2} / 5 \% \mathrm{CO}_{2} ; \mathrm{pH} 7.35$ ).

\subsubsection{Spinning disk confocal imaging}

QDs imaging in acute slices was performed in a Leica DMI6000 inverted microscope (Leica Microsystems) with a Yokogawa spinning disk unit CSU-X1. The setup was equipped with live cell chamber and temperature was constantly kept at $37^{\circ}$ C. EGFP transfections were excited with a 491-nm laser line and emission was observed with a standard GFP filter. For single QDs tracking, QDs were excited with a 561-nm diode laser line and the emission was filtered with a $650-$ to $800-\mathrm{nm}$ bandpass filter. QDs tracking images were obtained with $63 \times$ oil objective (Leica HCX PL APO 63x/1.40-060).

\subsection{Single Quantum Dots Tracking in Cultured Organotypic Brain Slices}

\subsubsection{Organotypic slice preparation}

Organotypic slice cultures were prepared as previously described. ${ }^{24}$ Briefly, 350- $\mu \mathrm{m}$-thick hippocampal slices were obtained from postnatal day 5 to 7 Sprague-Dawley rats using a McIlwain tissue chopper and were placed in a preheated $\left(37^{\circ} \mathrm{C}\right.$ ) dissection medium containing (in $\mathrm{mM}$ ): 175 sucrose, 25 D-glucose, $50 \mathrm{NaCl}, 0.5 \mathrm{CaCl} 2,2.5 \mathrm{KCl}, 0.66 \mathrm{KH} 2 \mathrm{PO} 4,2$ $\mathrm{MgCl} 2,0.28 \mathrm{MgSO} 4-7 \mathrm{H} 2 \mathrm{O}, \quad 0.85$ Na2HPO4-12H2O, 2.7 NaHCO3, 0.4 4-(2-hydroxyethyl)-1-piperazineethanesulfonic acid (HEPES), $2 \times 10^{-5} \%$ phenol red, and $\mathrm{pH} 7.3$ (all products from Sigma unless specified). After 25 min of incubation, slices were transferred on white FHLC membranes $(0.45 \mu \mathrm{m})$ set on Millicell cell culture inserts (Millipore, $0.4 \mathrm{~mm} ; \varnothing 30 \mathrm{~mm}$ ) and cultured for up to 14 days on multiwell plates at $35^{\circ} \mathrm{C} / 5 \% \mathrm{CO}_{2}$ in a culture medium composed of $50 \%$ basal medium Eagle, $25 \%$ Hank's balanced salt solution $1 \times$ (with $\mathrm{MgCl} 2 / \mathrm{CaCl} 2$ ), 
$25 \%$ heat-inactivated horse serum, $0.45 \%$ D-glucose, $1 \mathrm{mM} \mathrm{L}$ glutamine (all products from Gibco unless specified). The medium was changed every 2 to 3 days.

\subsubsection{Single-cell electroporation}

Electroporation of single pyramidal neurons was performed as previously described. ${ }^{25}$ Briefly, individual CA1 pyramidal neurons from 4 to 6 days in vitro (div) hippocampal slices were electroporated to transfect cDNA encoding GluN1-SEP and EGFP. Plasmids $(2.5 \mu \mathrm{l}$ each at $1 \mu \mathrm{g} / \mu \mathrm{l})$ were dissolved in a filtered cesium-based solution containing (in $\mathrm{mM}$ ): 135 cesium-methanesulfonate, $8 \mathrm{NaCl}, 10 \mathrm{HEPES}, 0.2$ ethylene glycol-bis( $\beta$-aminoethyl ether)-N,N,N',N'-tetraacetic acid, 4 $\mathrm{Na}_{2}$ ATP, 0.33 Na3GTP, 5 tetraethylammonium chloride, and $\mathrm{pH} 7.3$ (all products from Sigma unless specified). This solution was supplemented with $10 \mu \mathrm{l}$ of filtered endotoxin-free buffer TE (Qiagen), then centrifuged twice to pull down potential debris $\left(10,000 \mathrm{rpm}, 15 \mathrm{~min}, 4^{\circ} \mathrm{C}\right)$ and used to fill 5- to 6-M $\Omega$ borosilicate patch pipettes. Electroporation was performed in $2 \mathrm{ml}$ of prewarmed $\left(37^{\circ} \mathrm{C} / 5 \% \mathrm{CO}_{2}\right)$ HEPES-based ACSF containing (in mM): $130 \mathrm{NaCl}, 2.5 \mathrm{KCl}, 2.2 \mathrm{CaCl}_{2}, 1.5 \mathrm{MgCl}_{2}, 10$ HEPES, and 10 D-glucose. Plasmid transfer was allowed by the delivery of $50 \mu$ s-width square-pulses at $100 \mathrm{~Hz}$ (1-s duration; $-14-\mathrm{V}$ current amplitude).

\subsubsection{Quantum dots labeling and imaging}

Two to three days after electroporation, hippocampal brain slices were carefully detached from their culture membranes using a scalpel and incubated $\left(10 \mathrm{~min}, 37^{\circ} \mathrm{C}\right)$ with mouse anti-GFP antibodies (Molecular Probes) at a dilution of 1:5000 or GFP rabbit serum polyclonal antibody (Molecular Probes) at a dilution of $1: 10,000$ in HEPES-based ACSF containing (in $\mathrm{mM}$ ): $130 \mathrm{NaCl}, 2.5 \mathrm{KCl}, 2.2 \mathrm{CaCl}_{2}, 1.5 \mathrm{MgCl}_{2}, 10 \mathrm{HEPES}$, and 10 D-glucose. Slices were then washed and incubated $(10 \mathrm{~min}$, $37^{\circ} \mathrm{C}$ ) with QD655 goat $\mathrm{F}\left(\mathrm{ab}^{\prime}\right) 2$ antimouse or antirabbit IgG (Invitrogen) at a dilution of $1: 10,000$, respectively. Nonspecific binding was blocked by adding $1 \%$ bovine serum albumin (BSA) (Sigma) to the antibody and QDs solutions. QDs were detected using a mercury lamp, appropriate excitation/emission filters, and an electron multiplying charge-coupled device (EMCCD) camera (Evolve, Photometrics).

\subsubsection{N-Methyl-D-aspartate receptors cross-linking}

As previously described, ${ }^{26}$ for the cross-linking (x-link) experiments, organotypic slices were incubated with highly concentrated (1:5 dilution in HEPES-based ACSF) polyclonal antibodies directed against GFP (Molecular Probes) for $30 \mathrm{~min}$ at $35^{\circ} \mathrm{C}$. Incubation with the same concentration of monoclonal antiflag M2 (Sigma, \#F1804) was used as a control.

\subsection{Single Quantum Dots Tracking in Cultured Neurons}

\subsubsection{Primary cell culture}

Cultures of hippocampal neurons were prepared from E18 Sprague-Dawley rats as previously described. ${ }^{27}$ Briefly, cells were plated at a density of $3.5 \times 10^{5}$ cells per dish on polylysine precoated coverslips. Coverslips were maintained in a $3 \%$ horse serum-containing neurobasal medium (Invitrogen). After four div, the medium was replaced by serum-free neurobasal medium. Cultures were maintained at $37^{\circ} \mathrm{C}$ in $5 \%$ $\mathrm{CO}_{2}$ for $15 \mathrm{div}$ at maximum. Cultured hippocampal neurons were transfected with cDNA constructs coding for GluN1SEP and EGFP at 10 div using the Effectene kit (Qiagen).

\subsubsection{Quantum dots labeling and imaging}

Hippocampal neurons expressing GluN1-SEP were incubated $\left(10 \mathrm{~min}, 37^{\circ} \mathrm{C}\right)$ with mouse anti-GFP IgG1 $\kappa$ antibodies $(1: 5000$, Roche) or anti-GFP antibodies (1:10,000, Molecular Probes) in neurobasal medium (Invitrogen). Neurons were then washed and incubated $\left(10 \mathrm{~min}, 37^{\circ} \mathrm{C}\right)$ with QD655 goat $\mathrm{F}(\mathrm{ab}) 2$ antimouse $(1: 20000)$ or rabbit IgG $(1: 10,000)$ (Invitrogen), respectively. Nonspecific binding was blocked by adding $1 \%$ BSA (Sigma) to the antibody and QDs solutions. QDs were detected using a mercury lamp, appropriate excitation/emission filters and an EM-CCD camera (Evolve, Photometrics).

\subsection{Image acquisition and particle tracking}

Images were acquired using Metamorph software (Molecular Devices) with an acquisition time of $50 \mathrm{~ms}$ with up to 500 consecutive frames. Image stacks were analyzed using a custom program operating as a Metamorph plugin, based on wavelet segmentation for localization ${ }^{28,29}$ and simulated annealing algorithms for tracking. ${ }^{30,31}$ It allows both the localization of individual QD in all image plans and their reconnection through successive images. The instantaneous diffusion coefficient, $D$, was calculated for each trajectory from linear fits of the first four points of the mean-square displacement (MSD) function versus time.

\section{Results}

To perform single nanoparticle tracking in intact brain tissue and compare the lateral diffusion properties of NMDAR between different neuronal preparations, we overexpressed a recombinant version of the NMDAR obligatory GluN1 subunit bearing an SEP tag at its extracellular N-terminus in neurons from mixed primary hippocampal cultures, organotypic cultured hippocampal slices, and acute brain slices (cortex and hippocampus). As described in Fig. 1, different strategies were used to express exogenous GluN1-NMDAR, ranging from chemical transfection (e.g., calcium phosphate and lipid reagents) for cultured neurons to single-cell electroporation for cultured slices or whole brain electroporation (in utero or postnatal ${ }^{23}$ ) for acute slices applications. Once expressed in neurons, tagged receptors were targeted using a complex containing an antibody (IgG type) directed against the tag (i.e., GFP) and a single QD emitting at $655 \mathrm{~nm}$ (QD655). The coupling between IgG and QDs was performed by coating the QDs with Fab fragments directed against IgG Fc fragments. QDs were functionalized with antibodies by simple incubation at room temperature for $30 \mathrm{~min}$. The antibody-QDs complex was either bath-applied for cultured neurons and organotypic slices, or injected into the cerebroventricular system for acute brain slices. Intracerebroventricular injection resulted in a smooth distribution of QDs over the entire brain, minimizing the characteristic inflammation associated with local brain tissue injections. ${ }^{32}$ Rat pups (4 to 6 postnatal days old) were sacrificed 1.5 to $2 \mathrm{~h}$ after antibody-QDs injections, and acute brain slices were prepared as described in Sec. 2 [Fig. 1(c)]. Single QDs detection and tracking in cultures and organotypic preparations were performed using wide-field epifluorescence imaging, while spinning disk confocal microscopy 
(a)

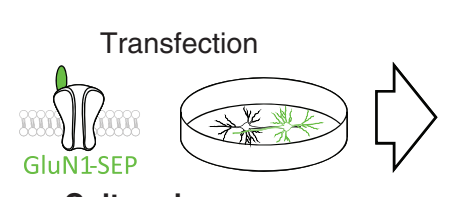

Cultured neurons

(b)
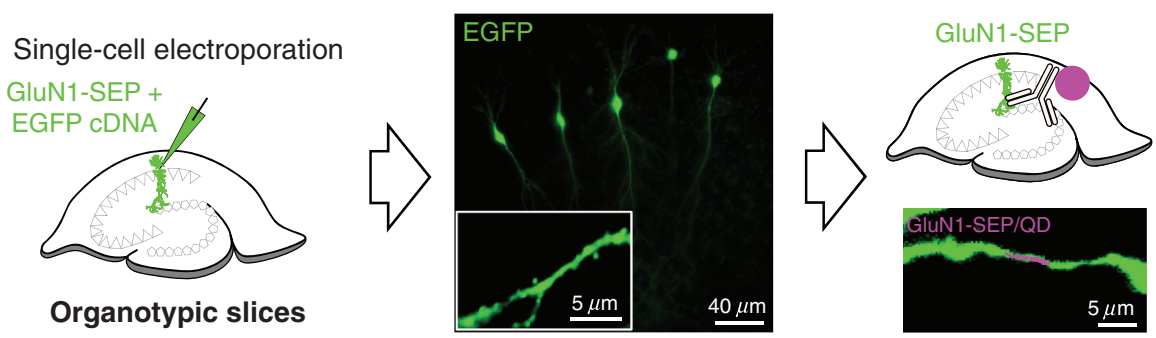

(c)

Postnatal electroporation
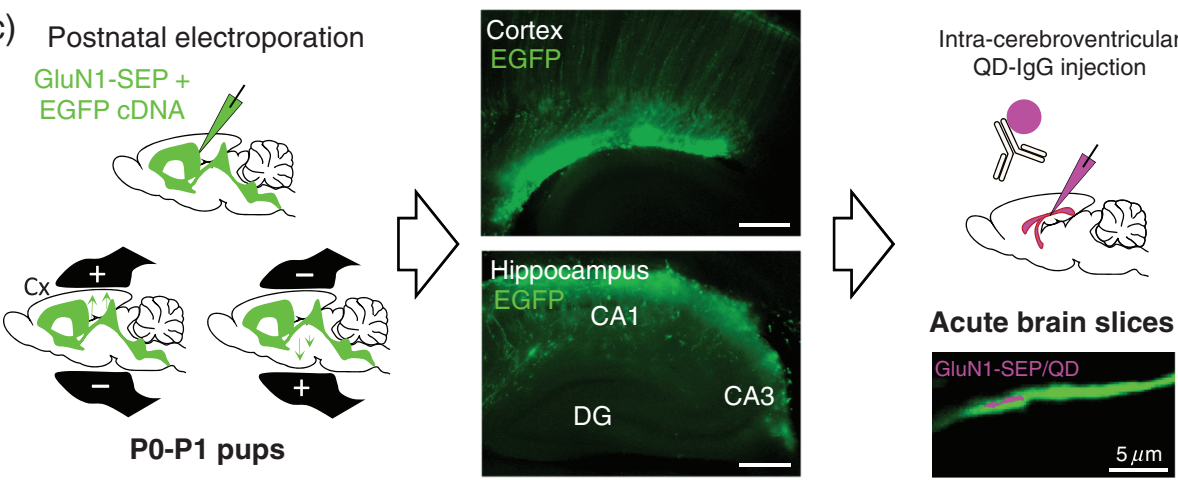

Acute brain slices

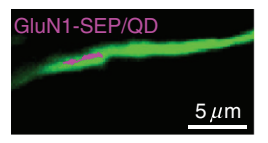

Fig. 1 Tracking of individual exogenous NMDAR in dissociated neuronal cultures, organotypic, and acute brain slices using QDs coupled to antibodies against GFP. (a) Cultured hippocampal neurons were transfected at 10 days in vitro to express recombinant GluN1-SEP and EGFP. Two to three days after transfection, cells were incubated with QDs functionalized with mouse polyclonal anti-GFP antibodies. (b) Individual CA1 pyramidal neurons from 4 to 6 days in vitro hippocampal slices were electroporated to express recombinant GluN1-SEP and EGFP. Two to three days after electroporation, organotypic slices were carefully detached from their inserts and incubated with QDs functionalized with antiGFP antibodies. (c) P0-P1 rats were injected in the cerebral ventricles with cDNAs coding for recombinant GluN1-SEP and EGFP, then electroporated using forceps-type circular electrodes to allow gene transfer to either hippocampal (Hipp.) or cortical (Cx) neurons. Three days after electroporation, pups received an intraventricular injection of QDs functionalized with anti-GFP antibodies before sacrifice and brain slice preparation. GluN1-SEP/QDs trajectories (magenta) at the surface of transfected or electroporated neurons (green) were recorded with an acquisition rate of $20 \mathrm{~Hz}$. Scale bar $=500 \mu \mathrm{m}$ (cortex), $300 \mu \mathrm{m}$ (hippocampus).

was favored for acute slices since it provides fast sampling rates ( $20 \mathrm{~Hz}$ in our experimental configuration) and minimizes out-offocus light. QDs detection in cultures was achieved at a high signal-to-noise ratio (SNR 20) while SNR was much lower in slices $(\sim 5)$, where the background noise is more pronounced because of light scattering, absorption, and tissue autofluorescence [Fig. 2(a)]. Single QDs were 2 to 4 pixels wide allowing to fit with a Gaussian curve and establish a pointing accuracy of about $30 \mathrm{~nm}$. The classical blinking property of QDs was also observed, allowing to ascertain that single and not multiple QDs were indeed resolved [Figs. 2(b) and 2(c)].

Our results show that the accessibility of tagged receptors is obviously different between cultured and intact brain structures. Differences in terms of extracellular space configuration and density-loose in cultured neurons but increasingly tighter in organotypic and acute brain slices-are expected to impact the capacity to properly wash out the antibody-QDs complexes in the most compact systems. To assess this, we calculated an enrichment factor in the three different preparations, which were determined as the number of QDs on GluN1-SEP/EGFP-transfected neurons versus the number of QDs in the background (both of which were normalized to the specific and unspecific volume, respectively). An enrichment factor of 1 would mean an even distribution of the particles, without any specificity of the antibody-QDs complexes for the transfected neurons. Enrichment factors in the three preparations ranged from 4 to 16, indicating that the anti-GFP antibody-QDs complexes were well enriched onto GFP-positive neurons in all conditions (Fig. 3). As expected, the enrichment factor measured in cultured neurons, in which the wash-in and wash-out processes are highly efficient, was significantly higher than for acute brain slices. In the latter preparation, a higher amount of off-target 

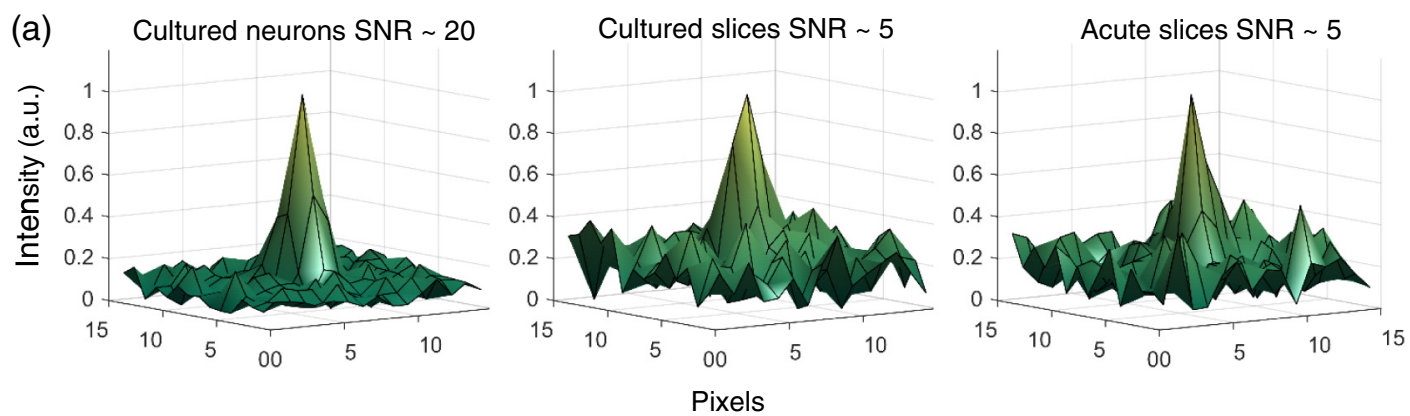

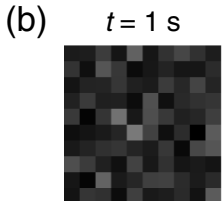

$t=4 \mathrm{~s}$
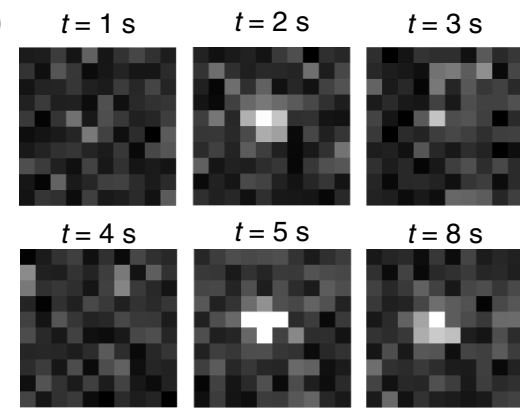

$t=8 \mathrm{~s}$

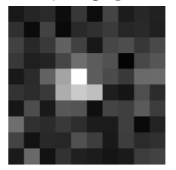

$2 \mu \mathrm{m}$

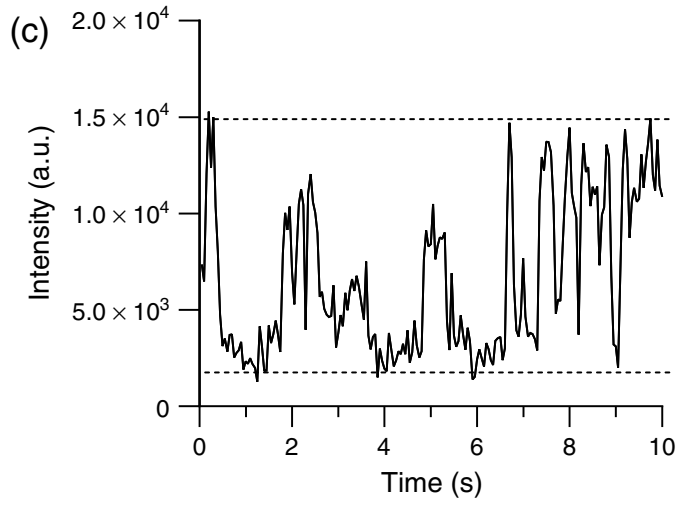

Fig. 2 QDs detection in different neuronal preparations. (a) Representative examples of SNR for individual QDs detection in cultured neurons (SNR 20), organotypic, and acute brain slices (SNR $\sim 5)$. (b) Time behavior of an individual GluN1-NMDAR-attached QDs in an acute brain slice showing blinking between bright and dark states, as illustrated by (c) the corresponding variations on the fluorescence intensity versus time plot.
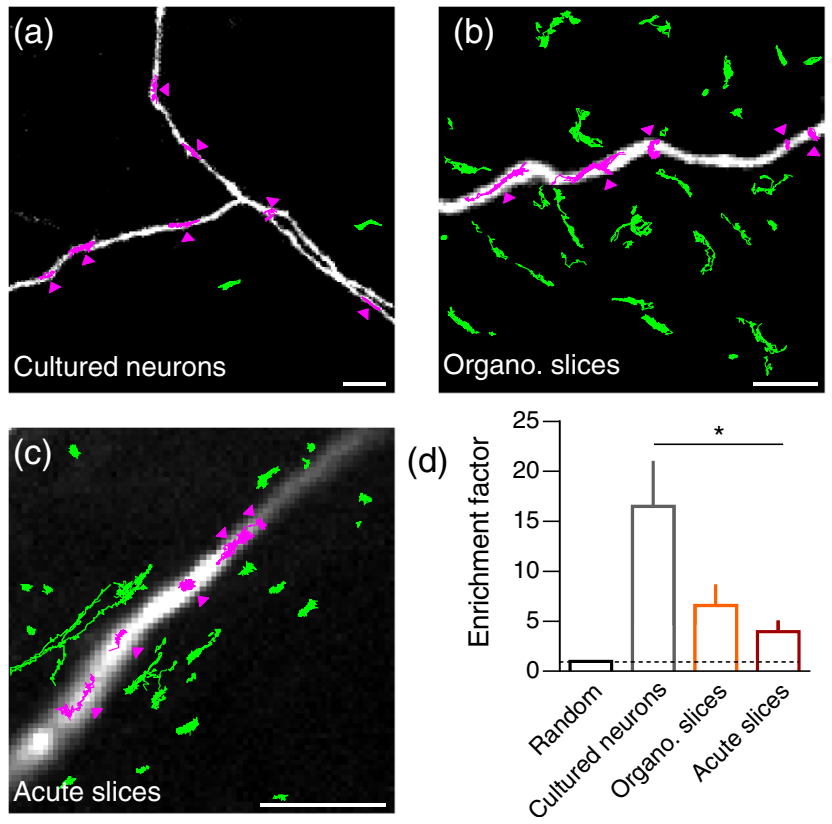

Fig. 3 Quantification of the relative enrichment of QDs labeling on GluN1-SEP/EGFP-transfected neurons in dissociated cultures, organotypic, and acute brain slices. Representative specific (magenta, arrow heads) and unspecific (green) QDs trajectories in (a) dissociated cultures, (b) organotypic, and (c) acute brain slices. Scale bars: $5 \mu \mathrm{m}$. (d) Quantification of the enrichment factor in the three experimental conditions, defined as the number of QDs on GluN1-SEP/EGFP-transfected neurons versus the number of QDs in the background (normalized to the specific and unspecific volume, respectively). Data were obtained from 10 neurons in 3 dissociated cultures, 13 neurons in 7 organotypic slice cultures, and 10 neurons in 7 acute slices, respectively.
antibody-QDs complexes was observed, likely due to a higher chance of in vivo internalization and/or retention in confined areas because of the tortuous complexity of the extracellular space in intact brain tissue. ${ }^{33}$ Noteworthy, the enrichment factor in these different preparations also depends on the nature of tagged receptors (e.g., amount at the cell surface, availability). Indeed, enrichment factors for GFP-tagged dopamine D1 receptors as measured in cultured neurons, organotypic and acute slices were found to be 174, 19, and 6.2 (data not shown), respectively, indicating an overall higher enrichment than for GluN1-NMDAR.

While unspecific and GFP signal-overlapping trajectories could not be distinguished based on length in either preparation (e.g., mean trajectory length in cultured organotypic slices: specific $=3.33 \mathrm{~s}, n=3253$ trajectories; unspecific $=3.41 \mathrm{~s}$, $n=46,096$ trajectories; $p=0.8$ ), they displayed very distinctive mobility patterns. We then quantified the diffusion characteristics of the complexes thanks to the use of a powerful and custom program operating as a Metamorph plugin, i.e., based on wavelet segmentation for localization ${ }^{28,29}$ and simulated annealing algorithms for tracking ${ }^{30}$ (see methods). The transfected neuron-associated trajectories were found to be characterized by significantly slower instantaneous diffusion coefficients (median instantaneous diffusion coefficient $\pm \mathrm{IQR}$ : specific, $D=$ $0.0448 \pm \mathrm{IQR} \quad 0.01$ to $0.124 \mu \mathrm{m}^{2} / \mathrm{s}, n=3,253$ trajectories; unspecific, $D=0.0671 \pm \mathrm{IQR} \quad 0.016$ to $0.067 \mu \mathrm{m}^{2} / \mathrm{s}$, $n=46,096$ trajectories; $* * * p<0.0001)$ and higher levels of confinement [Figs. 4(a)-4(c)], both of which allowed a clear separation with unspecific signals. Moreover, modulating receptor mobility through an antibody-based cross-linking strategy $^{30}$ selectively impacted the diffusion properties of 
(a)

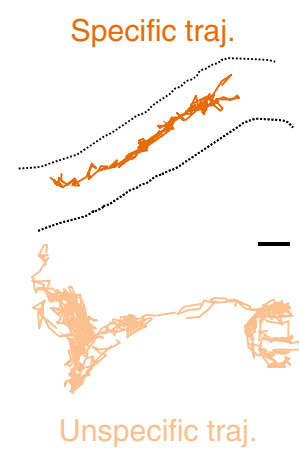

(d)
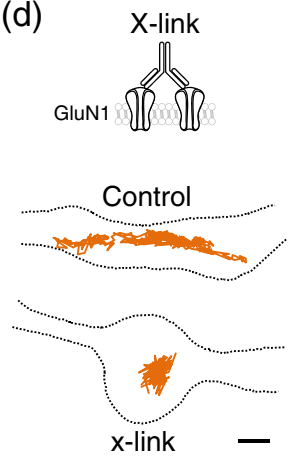

(b)

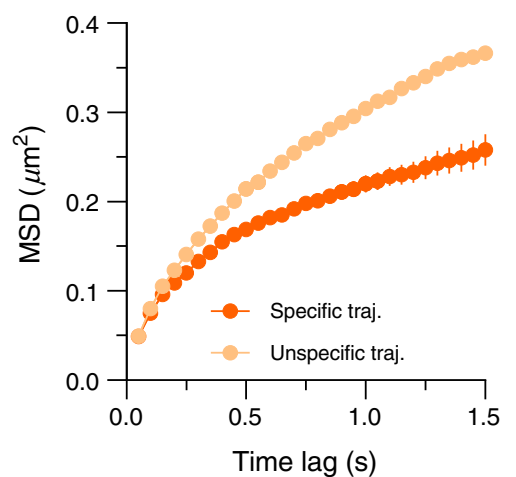

(e) 0.8

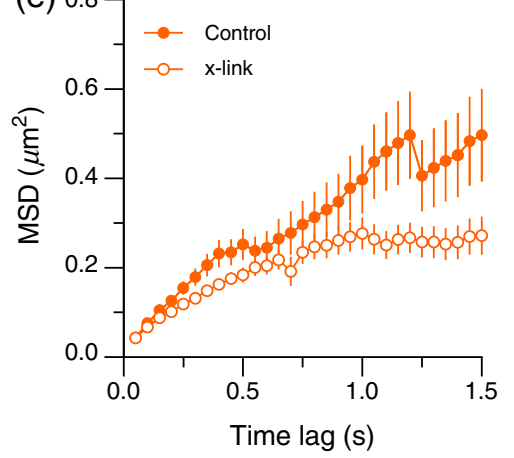

(c)

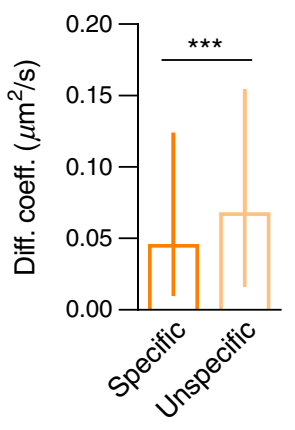

(f)

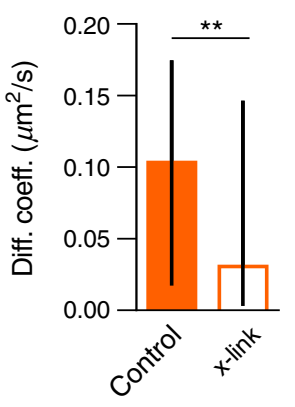

Fig. 4 Comparison of specific and unspecific trajectory properties in cultured slices. (a) Representative specific (orange) and unspecific (light orange) trajectories obtained from organotypic slices. Scale bar: $1 \mu \mathrm{m}$. (b) MSD versus time plot for specific (orange) and unspecific (light orange) trajectories. (c) Instantaneous diffusion coefficients for specific (orange) and unspecific (light orange) trajectories. Data are represented as median \pm IQR. (d) Representative trajectories obtained from organotypic slices in control and cross-link ( $x$-link) conditions (the $x$-link principle is displayed on the top of the panel). Scale bar: $1 \mu \mathrm{m}$. (e) MSD versus time plot for trajectories in control (control) and crosslink (x-link) conditions. (f) Instantaneous diffusion coefficients for trajectories in control and crosslink ( $\mathrm{x}$-link) conditions. Data are represented as median $\pm I Q R$.

GFP signal-overlapping QDs [Figs. 4(d) and 4(f)] (median instantaneous diffusion coefficient $\pm \mathrm{IQR}$ : specific control, $D=$ $0.1033 \pm$ IQR 0.017 to $0.175 \mu \mathrm{m}^{2} / \mathrm{s}, n=72$ trajectories; specific x-link, $D=0.0306 \pm \mathrm{IQR} 0.003$ to $0.146 \mu \mathrm{m}^{2} / \mathrm{s}, n=247$ trajectories; $* * p<0.01)$ whereas it had no influence on the dynamics of randomly distributed ones (median instantaneous diffusion coefficient $\pm \mathrm{IQR}$ : unspecific, $D=0.1117 \pm \mathrm{IQR}$ 0.030 to $0.21 \mu \mathrm{m}^{2} / \mathrm{s}, n=80$ trajectories; unspecific $\mathrm{x}$-link, $D=0.0688 \pm$ IQR 0.008 to $0.194 \mu \mathrm{m}^{2} / \mathrm{s}, n=250$ trajectories; $p=0.06$ ), suggesting that transfected neuron-associated fluorophores are specifically bound to SEP-tagged GluN1-NMDAR.

Importantly, GluN1-NMDAR trajectories overlapping the GFP signal of transfected neurons presented a wide range of behaviors, both in cultured and acute slices, ranging from immobile to slowly and highly mobile along dendrites and spines [Figs. 5(a) and 5(b)]. As previously reported in cultured neurons, ${ }^{15}$ single NMDAR were also found to rapidly alternate between slow and fast diffusion phases in cultured and acute slices [Figs. 5(c) and 5(d)], likely reflecting changing cellular environments explored by the receptor. We calculated the instantaneous diffusion coefficients of each trajectory as described in Sec. 2. When comparing the diffusion coefficients of GluN1NMDAR in the three systems, it emerged that receptor surface diffusion was slower in acute slices when compared with cultured preparations [Figs. 5(e) and 5(f)]. Noteworthy, the fractions of immobile receptors, defined as presenting diffusion coefficients lower than $0.005 \mu \mathrm{m}^{2} / \mathrm{s}$ [first point of the cumulative distribution; Fig. 5(e)], were similar for the three cases. The reduced diffusion in more intact preparations appear thus to be related to a relative increase in slowly mobile NMDAR. We tested whether this apparent reduction in NMDAR mobility results from an increased internalization of receptors. For this, cultured slices were exposed to dynasore $(80 \mu \mathrm{M})$ to block dynamin-dependent internalization pathways (including clathrin- and caveolin-ones). We report that NMDAR diffusion was unchanged by the treatment (median instantaneous diffusion coefficient $\pm \mathrm{IQR}$ : control, $D=0.0695 \pm \mathrm{IQR} 0.01$ to $0.173 \mu \mathrm{m}^{2} / \mathrm{s}, \quad n=379$ trajectories; dynasore, $D=0.07 \pm$ IQR 0.007 to $0.204 \mu \mathrm{m}^{2} / \mathrm{s}, n=161$ trajectories; $p=0.705$ ), suggesting that the internalization rate/process does not directly contribute to the observed diffusion parameters. The difference in mobility between preparations could however be a consequence of tracking receptors with two-dimensional (2-D) resolution in a three-dimensional (3-D) environment. Indeed, we observed a significant decrease in trajectory length when comparing 2-D dissociated cultures with 3-D cultured organotypic and acute brain slices (mean trajectory length \pm SEM: cultures, $l=11.2 \pm 0.5 \mathrm{~s}, n=350$ trajectories; organotypic slices, $l=$ $3.3 \pm 0.1 \mathrm{~s}, \quad n=3253$ trajectories; acute slices, $l=5.6 \pm$ $0.4 \mathrm{~s}, n=372$ trajectories; $* * * p<0.0001$ ), suggesting that receptors cannot be tracked over long periods of time in brain tissue [Fig. 5(f)]. This is likely due to the fact that mobile 

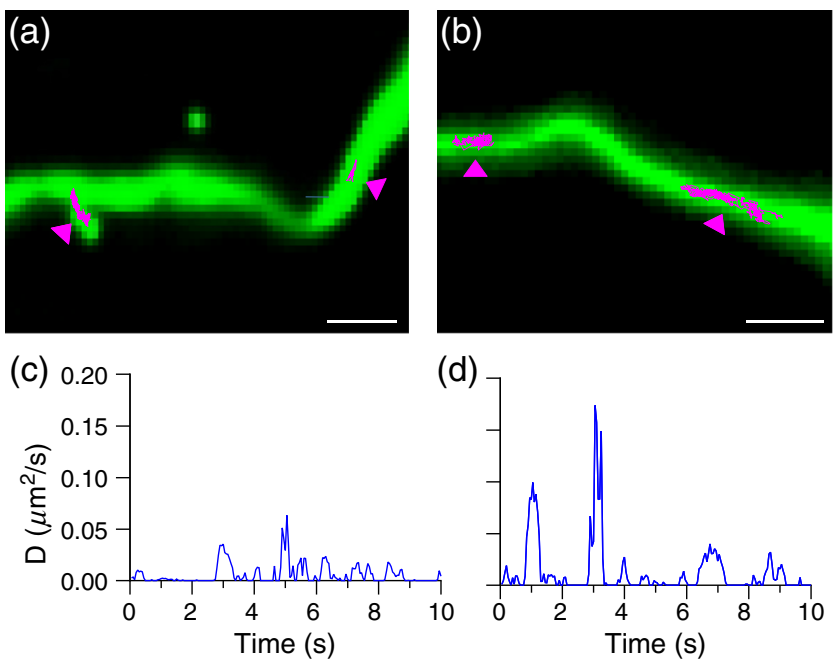

(d)


Fig. 5 Features of NMDAR diffusion in different brain preparations. Selection of trajectories in (a) acute and (b) cultured slices illustrating how GluN1-NMDAR diffuses along electroporated neurons. Scale bars: $2.5 \mu \mathrm{m}$. (c and d) Instantaneous diffusion coefficients calculated for each time-point of the selected trajectories from (c) acute and (d) cultured organotypic slices, showing how receptors alternate between mobile and immobile behaviors. (e) Cumulative distributions of the instantaneous diffusion coefficients of surface GluN1-NMDAR in cultured neurons (gray; $n=390$ trajectories), cultured organotypic (orange; $n=409$ ), and acute slices (red; $n=46$ ). The binning was set to $0.005 \mu \mathrm{m}^{2} / \mathrm{s}$ (the first point represents the immobile fraction). (f) Mean trajectory lengths in the different preparations.

receptor-QDs complexes go rapidly out of focus in a 3-D environment and are thus potentially underestimated, favoring the collection of slowly moving receptors. To note, the diffusion of GluN1-NMDAR in both acute and cultured slices was found to be slower than the diffusion of CFP-tagged recombinant dopamine D1 receptors tracked in acute brain slices in identical experimental conditions, in accordance with previous reports showing that D1 receptors are more diffusive than NMDAR in dissociated neuronal cultures. ${ }^{32}$ To further test whether the pattern of diffusing GluN1-NMDAR was different between cultured and acute brain slices, we randomly selected a subset of long GluN1-NMDAR trajectories ( $>400$ frames duration) in each condition and calculated their respective MSD. As shown in Fig. 6, initial phases of the MSD versus time plots were not different when comparing cultured and acute brain slices, suggesting that the pattern of GluN1-NMDAR surface exploration was relatively similar at short lag times in both preparations. However, the surface explored by receptors appeared to be significantly larger in cultured organotypic slices at longer lag times, indicating a higher level of receptor confinement in acute preparations [Fig. 6(b)].

One may notice that the MSD-based calculation of GluN1NMDAR trajectories is subject to statistical uncertainties due to the limited length of the trajectories. This complicates the (a)
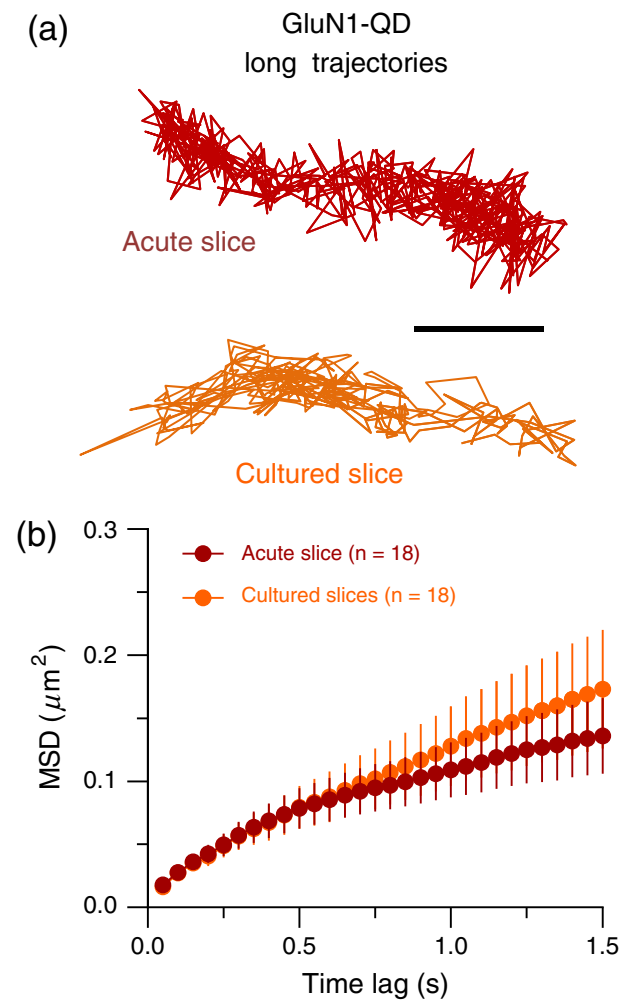

Fig. 6 MSD comparison for long trajectories. (a) GluN1-NMDAR-QDs trajectories from acute (red) and organotypic (orange) brain slices. Scale bar: $500 \mathrm{~nm}$. (b) MSD versus time plot for a subset of trajectories with over 400 frames in acute and cultured slices $(n=18$ trajectories in both conditions). The MSD shows a similar behavior at short lag times and a higher confinement in acute slices at longer lag times.

comparison of diffusion parameters that are potentially blurred by movements of different receptor subtypes. We thus performed an additional analysis to investigate the global mobility behaviors of receptor subpopulations,${ }^{28}$ regardless of trajectory length. It consisted of analyzing the probability distributions of the square displacements $r^{2}$ (denominated "squared steps") performed by GluN1-NMDAR during a given time interval $\tau$. For each time interval, we could fit these step distributions with three exponential decays, identifying three distinct receptor populations according to the obtained decay constants. Examples of the step distributions are shown for the time interval $\tau=0.25 \mathrm{~s}$ in Fig. 7(a), with the corresponding fit for the acute case [Fig. 7 (b)]. By fitting each step distribution with three exponential decay functions, the time constants obtained allowed a reconstruction of MSD plots for each receptor subpopulation [Fig. 7(c)]. As expected, the step distributions further indicate that the probability of having large displacements of GluN1NMDAR in acute brain slices is smaller than the probability of having large displacements in cultured slices [Fig. 7(a)], consistent with an overall reduced diffusion of surface GluN1NMDAR in acute preparations. Collectively, these data indicate that tagged GluN1-NMDAR can be tracked down at the surface of neurons in dissociated cultures, organotypic and acute slices, and that their overall dynamics decrease as a function of brain preparation complexity.

\section{Discussion}

Since the initial discovery of the lateral diffusion of glutamate receptors at the plasma membrane of neurons, ${ }^{14}$ NMDAR 
(a)

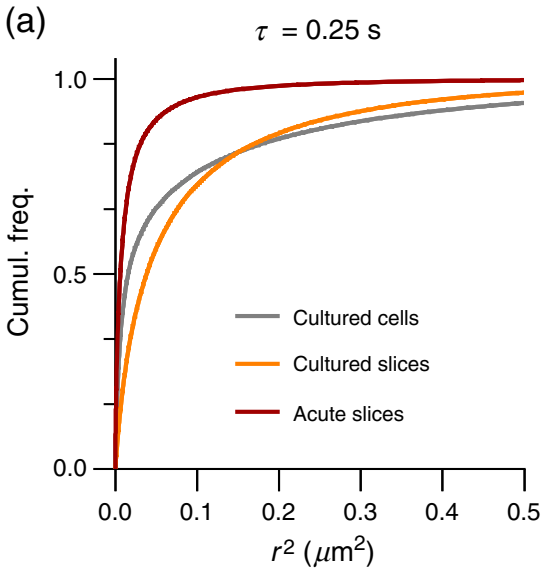

(b)

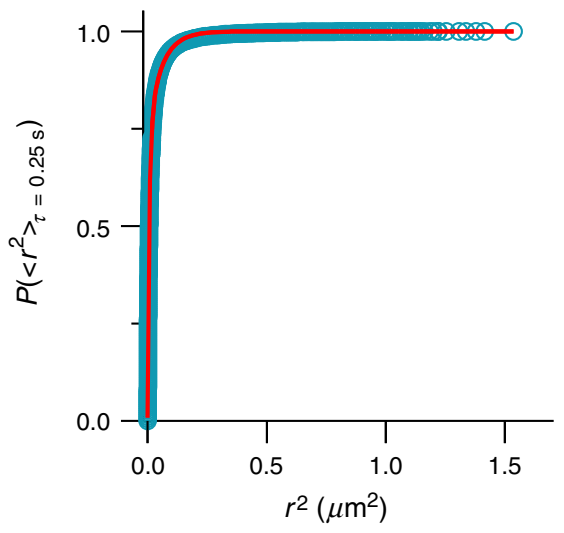

(c)

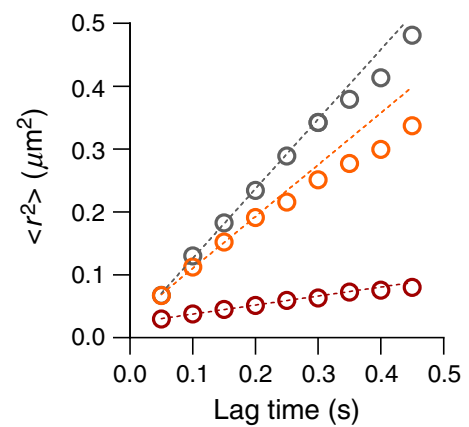

Slow subpopulation

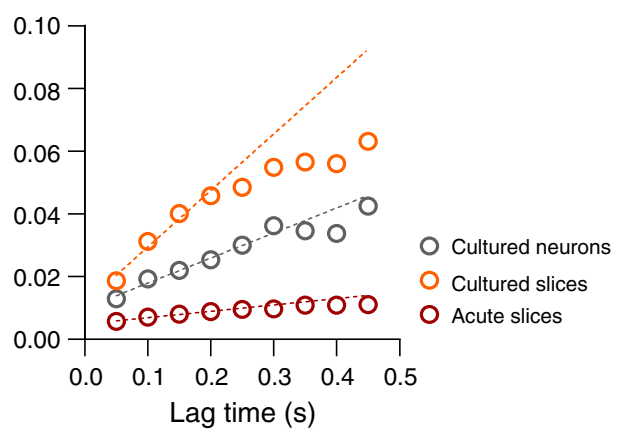

Fig. 7 Step size distribution analysis. (a) Squared step distributions for lag time $\tau=0.25 \mathrm{~s}$ in cultured neurons (gray), organotypic (orange), and acute slices (red). Measuring the displacement of each GluN1NMDAR-QDs complex for different lag times provides a statistically robust characterization of the dynamics and can account for heterogeneous populations. By fitting each distribution (a) with (b) triple exponential decay functions we can obtain (c) the time constants of each subpopulation.

surface dynamics have proved to play a key role in neuronal plasticity and memory formation. ${ }^{26,34}$ However, our current understanding of this process comes essentially from in vitro investigations based on primary cultures of neurons. Yet, key aspects of the regulation of NMDAR dynamics require intact brain preparations to be addressed, a challenge particularly obvious when considering the interplay between NMDAR and the extracellular environment. Indeed, NMDAR diffusion is affected by extracellular signaling proteins or matrix metalloproteases, ${ }^{35-37}$ suggesting a high level of regulation by components of the extracellular space. While interactions between NMDAR and the extracellular environment emerge as new actors in the physiology of NMDAR-related functions, most aspects of this interplay remain elusive by lack of adapted imaging approaches applicable to native brain preparations where it is preserved.

Here, we show that QDs tracking is a powerful tool to investigate diffusion of receptors in complex environments. It allowed us to track single NMDAR and confirm that they are mobile not only in cultured cells and slices but also in acute brain preparations where the tissue architecture is mostly preserved. Overall, NMDAR diffusion appeared to slow down as the brain preparations became more complex, although the fractions of immobile NMDAR are similar and highly mobile subpopulations of receptors are clearly observed in all preparations. Noteworthy, we previously reported similar diffusion values for GluN2B-NMDAR comparing in vitro and ex vivo samples with fluorescence recovery after photobleaching approaches, ${ }^{26}$ which contrasts with the observations made here on GluN1NMDAR which were found to be less diffusive in acute brain slices. While the nature of NMDAR subunits tracked is unlikely to account for this apparent discrepancy, this finding suggests that careful consideration must be taken when comparing diffusion measurements evaluated with ensemble and singleparticle imaging methods. In particular, a potential limitation of single-particle tracking with 2-D resolution in the 3-D environment of brain slices is the inability to perform long-time monitoring of highly mobile receptors that can rapidly move in and out of focus, possibly leading to an underestimation of the highly mobile populations. This hypothesis is supported by our observation that trajectory lengths are significantly decreased in slice preparations compared to dissociated cultures, which could result from this limitation. However, all the classical features of diffusing membrane molecules were recapitulated ex vivo, including well-characterized changes in diffusion modes for single NMDAR, which rapidly switched from immobile to diffusive states and back. To conclude, although the approach described here is still currently restricted to recombinant neurotransmitter receptors, assessing NMDAR surface dynamics ex vivo will significantly improve our understanding of the mechanisms controlling NMDAR-dependent cognitive functions, and likely shed new lights on the nanoscale impairments that affect receptor signaling in neurological and psychiatric diseases. 


\section{Acknowledgments}

This work was supported by the Centre National de la Recherche Scientifique (CNRS), Agence Nationale de la Recherche, Conseil Régional d'Aquitaine, and Marie Curie Individual Fellowship No. 326442. We would like to greatly thank J. B. Sibarita for providing us the analysis software for single-molecule localization and tracking. We also thank the Bordeaux Imaging Center (service unit of the CNRSINSERM and Bordeaux University, member of the National Infrastructure France BioImaging). The help of Christel Poujol and Sébastien Marais from Bordeaux Imaging Center is particularly acknowledged. We would like to thank Laurent Cognet and lab members for constructive discussion.

\section{References}

1. M. Maglione and S. J. Sigrist, "Seeing the forest tree by tree: superresolution light microscopy meets the neurosciences," Nat. Neurosci. 16, 790-797 (2013).

2. Z. Liu, L. D. Lavis, and E. Betzig, "Imaging live-cell dynamics and structure at the single-molecule level," Mol. Cell 58, 644-659 (2015).

3. B. Huang, M. Bates, and X. Zhuang, "Super-resolution fluorescence microscopy," Аnnu. Rev. Biochem. 78, 993-1016 (2009).

4. L. Cognet, B. Lounis, and D. Choquet, "Tracking receptors using individual fluorescent and nonfluorescent nanolabels," Cold Spring Harb. Protoc. 2014, 207-213 (2014).

5. L. Ladepeche, J. P. Dupuis, and L. Groc, "Surface trafficking of NMDA receptors: gathering from a partner to another," Semin. Cell Dev. Biol. 27C, 3-13 (2014).

6. A. Triller and D. Choquet, "New concepts in synaptic biology derived from single-molecule imaging," Neuron 59, 359-374 (2008).

7. M. Dahan et al., "Diffusion dynamics of glycine receptors revealed by single-quantum dot tracking," Science 302, 442-445 (2003).

8. G. Giannone et al., "Dynamic superresolution imaging of endogenous proteins on living cells at ultra-high density," Biophys. J. 99, 1303-1310 (2010).

9. E. Betzig et al., "Imaging intracellular fluorescent proteins at nanometer resolution," Science 313, 1642-1645 (2006).

10. X. Michalet et al., "Quantum dots for live cells, in vivo imaging, and diagnostics," Science 307, 538-544 (2005).

11. D. Choquet and A. Triller, "The dynamic synapse," Neuron 80, 691703 (2013).

12. L. Groc and D. Choquet, "Measurement and characteristics of neurotransmitter receptor surface trafficking (Review)," Mol. Membr. Biol. 25, 344-352 (2008)

13. S. Levi, M. Dahan, and A. Triller, "Labeling neuronal membrane receptors with quantum dots," Cold Spring Harb. Protoc. 2011, prot5580 (2011).

14. L. Groc et al., "Differential activity-dependent regulation of the lateral mobilities of AMPA and NMDA receptors," Nat. Neurosci. 7, 695-696 (2004).

15. L. Groc et al., "NMDA receptor surface mobility depends on NR2A-2B subunits," Proc. Natl. Acad. Sci. U.S.A. 103, 18769-18774 (2006).

16. L. Ladepeche et al., "Single-molecule imaging of the functional crosstalk between surface NMDA and dopamine D1 receptors," Proc. Natl. Acad. Sci. U.S.A. 110, 18005-18010 (2013).
17. H. Bannai et al., "Activity-dependent tuning of inhibitory neurotransmission based on GABAAR diffusion dynamics," Neuron 62, 670682 (2009).

18. J. Eriksen et al., "Visualization of dopamine transporter trafficking in live neurons by use of fluorescent cocaine analogs," J. Neurosci. 29, 6794-6808 (2009).

19. C. Murphy-Royal et al., "Surface diffusion of astrocytic glutamate transporters shapes synaptic transmission," Nat. Neurosci. 18, 219-226 (2015).

20. P. Paoletti, C. Bellone, and Q. Zhou, "NMDA receptor subunit diversity: impact on receptor properties, synaptic plasticity and disease," Nat. Rev. Neurosci. 14, 383-400 (2013).

21. C. G. Lau and R. S. Zukin, "NMDA receptor trafficking in synaptic plasticity and neuropsychiatric disorders," Nat. Rev. Neurosci. 8, 413-426 (2007).

22. B. Biermann et al., "Imaging of molecular surface dynamics in brain slices using single-particle tracking," Nat. Commun. 5, 3024 (2014).

23. H. Ito et al., "Establishment of an in vivo electroporation method into postnatal newborn neurons in the dentate gyrus," Hippocampus 24, 1449-1457 (2014).

24. L. Stoppini, P. A. Buchs, and D. Muller, "A simple method for organotypic cultures of nervous tissue," J. Neurosci. Methods 37, 173-182 (1991).

25. K. Haas et al., "Single-cell electroporation for gene transfer in vivo," Neuron 29, 583-591 (2001).

26. J. P. Dupuis et al., "Surface dynamics of GluN2B-NMDA receptors controls plasticity of maturing glutamate synapses," EMBO J. 33, 842-861 (2014).

27. L. Mikasova et al., "Disrupted surface cross-talk between NMDA and ephrin-B2 receptors in anti-NMDA encephalitis," Brain 135, 16061621 (2012).

28. I. Izeddin et al., "Wavelet analysis for single molecule localization microscopy," Opt. Express 20, 2081-2095 (2012).

29. A. Kechkar et al., "Real-time analysis and visualization for single-molecule based super-resolution microscopy," PLoS One 8, e62918 (2013).

30. V. Racine et al., "Multiple-target tracking of 3D fluorescent objects based on simulated annealing," in IEEE Int. Symp. on Biomedical Imaging, pp. 1020-1023 (2006).

31. J. B. Sibarita, "High-density single-particle tracking: quantifying molecule organization and dynamics at the nanoscale," Histochem. Cell Biol. 141, 587-595 (2014).

32. J. A. Varela et al., "Targeting neurotransmitter receptors with nanoparticles in vivo allows single-molecule tracking in acute brain slices," Nat. Commun. 7, 10947 (2016).

33. E. Sykova and C. Nicholson, "Diffusion in brain extracellular space," Physiol. Rev. 88, 1277-1340 (2008).

34. M. Potier et al., "Temporal memory and its enhancement by estradiol requires surface dynamics of hippocampal CA1 $N$-methyl-D-aspartate receptors," Biol. Psychiatry 79(9), 735-745 (2015).

35. L. Groc et al., "NMDA receptor surface trafficking and synaptic subunit composition are developmentally regulated by the extracellular matrix protein Reelin," J. Neurosci. 27, 10165-10175 (2007).

36. P. Michaluk et al., "Matrix metalloproteinase- 9 controls NMDA receptor surface diffusion through integrin beta1 signaling," J. Neurosci. 29, 6007-6012 (2009).

37. T. Papouin et al., "Synaptic and extrasynaptic NMDA receptors are gated by different endogenous coagonists," Cell 150, 633-646 (2012).

Biographies for the authors are not available. 